\title{
Reparación de la insuficiencia tricuspídea: resultados a largo plazo en 114 pacientes
}

\author{
Juan Carlos Bahamondes, Rodrigo Godoy, Abelardo Silva, Andrés Díaz, Mauricio Peña*. \\ ${ }^{\star}$ Enfermero Universitario. \\ Servicio de Cirugía Cardiovascular, Hospital Regional Temuco, Chile \\ Departamento de Cirugía, Universidad de La Frontera. \\ Temuco, Chile
}

Antecedentes: la reparación de la insuficiencia tricuspídea funcional está indicada en casos de insuficiencia cardíaca, generalmente, asociada a enfermedades de las válvulas cardíacas izquierdas y en algunos casos de enfermedad coronaria o cardiopatías congénitas.

Objetivo: evaluar los resultados a largo plazo de la reparación tricuspídea en pacientes operados en la región centro sur de Chile.

Pacientes y métodos: estudio retrospectivo de registros clínicos y operatorios en 114 pacientes con edad promedio 57.8 (DE 13) años (72 mujeres) sometidos a reparación tricuspídea asociada a otros procedimientos quirúrgicos entre 2009 y 2017.

Resultados: la etiología de la insuficiencia tricuspídea fue debida a enfermedad reumática inactiva en $45 \%$ y a endocarditis infecciosa en $2.6 \% .63 \%$ estaban en fibrilación auricular y $75 \%$ en capacidad funcional NYHC III. 15.8\% tenían cirugía valvular cardíaca pre- via. El ecocardiograma mostró insuficiencia severa en $56 \%$ de los casos y había hipertensión pulmonar severa en 39.5\%. La cirugía consistió en la implantación de un anillo semi-rígido en forma concomitante a reemplazo o reparación de lesión valvular mitral, reemplazo valvular aórtico, cierre de comunicación interauricular, cirugía coronaria o resección de tumor intra cardíaco. La mortalidad post operatoria global fue $16 \%$, debido a falla multisistémica en $6 \%$, insuficiencia cardíaca en $5 \%$ y hemorragia cerebral en $4.3 \%$. El seguimiento promedio fue 78.8 (DS 7.2) meses. La supervivencia actuarial fue $74 \%$ a los 60 y $68 \%$ a los 96 meses.

Conclusión: La cirugía de reparación de la insuficiencia tricuspídea moderada o severa, asociada a otras enfermedades cardíacas provee una buena recuperación sintomática, con excelente sobrevida alejada.

Palabras claves: Regurgitación tricuspídea, enfermedad valvular cardíaca, anuloplastía valvular. 


\section{Repair of tricuspid valve insufficiency: long-term results in 114 patients}

Background: Surgery for functional tricuspid valve insufficiency is indicated in cases of congestive heart failure usually associated to left heart valve diseases or as a concomitant surgery for other causes such as congenital or coronary disease.

Aim: To assess and report the long-term results of tricuspid valve repair in adult patients in a regional cardiac surgery center in south Chile.

Patients and Methods: Retrospective review of clinical and operative records of 114 patients aged $57.8+/-13$ years ( 72 women) subjected to tricuspid reparative surgery concomitant with other cardiac procedures between 2009 to 2017 .

Results: In $45 \%$ of cases etiology was due to inactive rheumatic disease and $2.6 \%$ was due to endocarditis. $63 \%$ presented with atrial fibrillation and $75 \%$ were in NYHC CFIII. $15.8 \%$ had a previous cardiac valve surgery. Preoperative echocardiography showed severe tricuspid insufficiency in $56 \%$ of cases and pulmonary hypertension was severe in $39.5 \%$. In all cases tricuspid repair was performed through the insertion of a semi rigid ring as a concomitant procedure for mitral repair/replacement in most cases, aortic valve replacement, surgical closure of an ASD, CABG surgery and the resection of cardiac tumors. Overall postoperative mortality was $16 \%$ due to multi-organic dysfunction in $6 \%$, cardiac failure in 5\% and cerebral hemorrhage in $4.3 \%$. Mean long term follow up was $78.8+/-7.2$ months. Actuarial survival was $74 \%$ at 60 and $68 \%$ at 96 months.

Conclusion: Surgical tricuspid valve repair for moderate to severe tricuspid insufficiency isolated or associated to other cardiac diseases provides a good symptomatic recovery, with an excellent long term survival.

Keywords: Tricuspid regurgitation (Mesh), tricuspid insufficiency (Mesh), heart valve disease (Mesh), heart valve annuloplasty (Mesh). 


\section{Introducción:}

La prevalencia de insuficiencia tricuspídea (IT) funcional es alta, y no tratada agrega una elevada morbilidad y mortalidad en el largo plazo ${ }^{1}$. Hasta hace un tiempo, se esperaba que la IT moderada o de menor grado, secundaria a existencia de patología valvular degenerativa izquierda, disminuyera o desapareciera después de la corrección quirúrgica de la valvulopatía causante ${ }^{2}$. Bajo esta mirada, la válvula tricúspide se trataba en forma conservadora, aunque algunos autores, en los años 70 , recomendaban abordar la quirúrgicamente la IT funcional secundaria a otras patologías cuando existía dilatación anular importante, mediante sutura del anillo tricuspídeo. Para ello, se desarrollaron varias técnicas, siendo la más conocida la técnica de De Vega ${ }^{3}$. Hoy en día, la cirugía de la IT funcional está indicada en casos de insuficiencia cardiaca asociada a lesiones valvulares izquierdas, sin aumentar el riesgo operatorio. Se ha demostrado que se produce remodelación del ventrículo derecho y mejoría de la capacidad funcional aun en ausencia de IT importante, cuando existe dilatación anular 4, 5, 6, 7, 8, 9, 10. Así también, la anuloplastia tricuspídea se puede realizar como procedimiento concomitante a cardiopatías congénitas y/o enfermedad coronaria avanzada Incluso puede efectuarse en el corazón donante para trasplante, existiendo evidencia en la literatura médica mundial que respalda la reparación tricuspídea con anillo ${ }^{11,12,13}$. Ello conlleva mejores resultados quirúrgicos a largo plazo, en comparación con la técnica de De Vega, por lo que ésta debiera quedar en el pasado $4,5,14,15$.

El objetivo del presente trabajo fue evaluar los resultados a largo plazo de la anuloplastia tricuspídea en pacientes adultos operados en el sur de Chile por insuficiencia tricuspídea moderada a severa.

\section{Pacientes y Método:}

Estudio descriptivo de 114 pacientes con diagnóstico de IT funcional moderada o severa sometidos a cirugía reparativa de la válvula tricuspídea concomitante a otros procedimientos cardiacos, en el periodo comprendido entre Marzo de 2009 y Septiembre de 2017, por el equipo de cirugía cardiovascular del Hospital Regional de Temuco. Se excluyeron del estudio a los pacientes con diagnóstico de IT leve en quienes no se efectuó la reparación valvular. Previo a la cirugía, todos los pacientes firmaron la solicitud de consentimiento informado. La revisión posterior de la información no significó nuevas intervenciones a los pacientes y se obvió, por tanto, una nueva aprobación institucional del Comité de ética.
Los datos fueron obtenidos de fichas clínicas, base de datos de Cirugía Cardiovascular y de protocolos operatorios. El seguimiento a largo plazo se realizó mediante controles clínicos en policlínico de cirugía cardíaca o de las consultas de médicos tratantes y contactos telefónicos. Además, se objetivó fecha y causa de muerte en el registro civil.

Se evaluaron características biodemográficas preoperatorias, patologías cardiovasculares concomitantes, la capacidad funcional $(\mathrm{CF})$ según la New York Heart Association (NYHA) y los hallazgos ecocardiográficos considerando el grado de IT, dilatación anular, la fracción de eyección del ventrículo izquierdo (FEVI) y la presencia de hipertensión pulmonar (HTP) clasificada como leve, moderada o severa. Se describe el tipo de cirugía concomitante efectuada junto con la anuloplastía tricuspídea. Se utilizó mayoritariamente el anillo incompleto de Carpentier-Edwards MC3 y en los últimos años el anillo Physio tricuspídeo, del mismo fabricante. Se analizaron las principales complicaciones postoperatorias ocurridas, como mortalidad a 30 días, infarto miocárdico, accidente cerebrovascular, infección de herida esternal, necesidad de instalación de marcapasos definitivo y sangrado postoperatorio que requiriera de exploración quirúrgica. En el seguimiento a largo plazo se determinó fecha y causa de muerte alejada, capacidad funcional, reaparición de disnea, y la necesidad de un nuevo procedimiento quirúrgico. Además, se describen los hallazgos encontrados en la ecocardiografía de control.

Técnica quirúrgica: Durante el período señalado, 4022 pacientes fueron intervenidos por patologías cardiovasculares, correspondiendo esta serie al $2.8 \%$ del total de operaciones en ese período. En todos los pacientes se accedió al corazón mediante esternotomía media clásica, debido a la mejor exposición del campo operatorio y por permitir una reparación exitosa de diferentes patologías cardiacas. Todos los pacientes fueron intervenidos con circulación extracorpórea, cardioplegia hemática fría administrada por vía anterógrada, retrógrada y/o ambas, hipotermia moderada $32^{\circ} \mathrm{C}-35^{\circ} \mathrm{C}$ y magnificación óptica de 2,5-3,0x.

Estadística: Se realizó una estadística descriptiva con medidas de tendencia central y dispersión y se utilizó el método de Kaplan-Meier para el cálculo de supervivencia actuarial con el programa computacional Stata $14.0^{\circledR}$. 


\section{Resultados:}

La edad promedio fue de 57,8 +- 13 años, el 63,2\% (72) de sexo femenino. El Euroscore II logístico promedio fue 6,2\% (2,2-29.7). En relación con la CF, 70\% de los pacientes estaba en CF III, 25\% en CF II, 3\% en CF IV y 2\% en CF I. El 98,2\% de los pacientes se presentó con insuficiencia cardiaca congestiva, y hubo diversas patologías asociadas como se señala en la Tabla 1.

\begin{tabular}{|c|c|c|}
\hline & $\mathbf{n}$ & $\%$ \\
\hline Insuficiencia cardíaca & 112 & 98.2 \\
\hline Fibrilación auricular & 72 & 63.2 \\
\hline Hipertensión & 59 & 51.8 \\
\hline Etiología reumática & 50 & 43.9 \\
\hline Diabetes & 22 & 19.3 \\
\hline Cirugía previa & 18 & 15.8 \\
\hline Dislipidemia & 10 & 8.8 \\
\hline Insuficiencia renal & 9 & 7.9 \\
\hline Enfermedad Coronaria & 7 & 6.1 \\
\hline Tabaquismo & 7 & 6.1 \\
\hline Accidente vascular encefálico & 6 & 5.3 \\
\hline Enfermedad pulmonar obstructiva & 4 & 3.5 \\
\hline
\end{tabular}

En el estudio ecocardiográfico preoperatorio, efectuado en todos los pacientes, se encontró que la IT fue catalogada como severa en $56 \%$ y moderada en $44 \%$. Se demostró dilatación del anillo tricuspídeo mayor a $40 \mathrm{~mm}$ en $65,7 \%$, la FEVI promedio fue $33,3 \%$ (rango $19-60 \%$ ). El 26,3\% presentó HTP moderada y en el 56,1\% esta fue catalogada como severa.

En relación con la cirugía efectuada, en todos los pacientes se utilizó un anillo incompleto semirrígido, procurando mantener la forma y anatomía de la válvula tricúspide, suturando este al anillo nativo con puntos separados de poliéster trenzado irreabsorbible, permitiendo así asegurar una reparación tricuspídea duradera y efectiva.

Se efectuaron diversos procedimientos asociados a la reparación tricuspídea, especialmente reemplazos valvulares mitrales o aórticos, como también reemplazos bivalvulares y cirugía coronaria. El detalle de ellos se indica en la Tabla 2. El tiempo promedio de circulación extracorpórea fue 101 minutos ( 35 - 228 minutos) y el tiempo promedio de clamp aórtico fue 75,1 minutos (32 -171 minutos).

Por otra parte, al evaluar la CF preoperatoria encontramos que la mayoría de los pacientes presentaban una $\mathrm{CF}$ III a IV al momento de la cirugía, lo cual demuestra su
Tabla 2. Cirugía asociada a la reparación tricuspídea

$\begin{array}{lc} & \% \\ \text { Reemplazo valvular mitral } & 56 \\ \text { Reemplazo valvular mitral y aórtico } & 19 \\ \text { Reparación de comunicación interauricular } & 7 \\ \text { Reemplazo valvular aórtico } & 6 \\ \text { Reemplazo valvular mitral y bypass aorto-coronario } & 5 \\ \text { Cirugía coronaria } & 4 \\ \text { Reemplazo mitral y resección de mixoma auricular } & 2 \\ \text { Reparación de prolapso mitral } & 1\end{array}$

compromiso clínico y la premura en efectuar la resolución quirúrgica. Este subgrupo presentó la mayor mortalidad de la serie, $68,5 \%$, en tanto que la mortalidad en el total de la serie fue $11,5 \%$. La mortalidad global fue de $8.8 \%$ a 30 días, la cual estuvo relacionada a pacientes con Euroscore mas alto, ocurriendo principalmente en los primeros 15 días posteriores a la cirugía $(60 \%)$. Las causas de mortalidad se detallan en la Tabla 3.

\section{Tabla 3. Mortalidad operatoria y causas de mortalidad}

$\begin{array}{lc} & \% \\ \text { Mortalidad operatoria } & 8.8 \\ \text { Causas } & \\ \text { Insuficiencia cardíaca } & 50 \\ \text { Accidente vascular encefálico } & 20 \\ \text { Falla multiorgánica } & 20 \\ \text { Hemorragia/disyunción aurículo-ventricular } & 10\end{array}$

En el periodo observado, $8.8 \%$ de los pacientes fue reintervenido debido a sangrado importante en el post operatorio inmediato, $5.3 \%$ desarrolló falla renal aguda que requirió diálisis, $2.3 \%$ presentó un accidente cerebral y el $0.9 \%$ de los pacientes tuvo una infección esternal profunda debido a germen nosocomial. Este fue tratado en forma agresiva con aseos quirúrgicos frecuentes, antibióticos y cierre de la pared torácica mediante la rotación de colgajos de músculo pectoral mayor, logrando una evolución satisfactoria. El $26.3 \%$ desarrolló un trastorno del ritmo cardiaco que requirió la instalación de un marcapasos definitivo lo que fue efectuado sin incidentes antes del alta hospitalaria de los pacientes.

Seguimiento. El seguimiento a largo plazo fue completo para 90 pacientes, con un promedio fue de 78,8 (DE \pm $7,2)$ meses. Al momento del cierre del estudio se evaluó 


\begin{tabular}{lr|}
$\begin{array}{l}\text { Tabla 4. Complicaciones de la Reparación de Insuficiencia } \\
\text { tricuspídea }\end{array}$ \\
\\
\\
Implantación de marcapasos & $\%$ \\
Re-operación & 26.3 \\
Insuficiencia renal & 8.8 \\
Accidente vascular encefálico & 5.3 \\
Infarto miocárdico & 2.6 \\
Infección esternal profunda & 0.9 \\
& 0.9 \\
\hline
\end{tabular}

la $\mathrm{CF}$ en el postoperatorio alejado, encontrándose que 95\% de los pacientes estaban en CF I y $5 \%$ en CF III, correspondiendo el resto a CF II. La probabilidad de supervivencia actuarial post cirugía fue $74 \%$ a 60 meses y de $68 \%$ a 96 meses de seguimiento (Figura 1).

Durante el seguimiento alejado se logró efectuar un control ecocardiográfico en 72 pacientes. Se encontró que $82 \%$ de los pacientes no tenían IT, $12,5 \%$ esta era leve y en $5.5 \%$ era moderada. La FEVI promedio fue $54.4 \%$, con una presión sistólica de arteria pulmonar menor a 31 mmHg en 68 pacientes, y entre 31 a $55 \mathrm{mmHg}$ en 4 pacientes. La función ventricular del ventrículo derecho no se evaluó, pero clínicamente no hubo signos de insuficiencia del mismo.

\section{Discusión:}

La reparación quirúrgica de la IT moderada y/o severa asociada a otros procedimientos cardiacos provee una excelente recuperación sintomática con muy buenos resultados en términos de supervivencia actuarial en el largo plazo $16,17,18,19,20,21,22,23$.

Nuestro estudio demuestra que la estrategia de reparación de rutina de la IT funcional moderada a severa, o cuando la dilatación anular es significativa en pacientes con enfermedades degenerativas concomitantes, prácticamente, elimina la IT sin aumentar el riesgo quirúrgico en forma importante. Por otra parte, la literatura indica que la reparación de la válvula tricúspide alcanza resultados superiores, no sólo en términos de supervivencia libre de insuficiencia residual o recurrente y síntomas, sino que demuestra una mejoría en la función ventricular derecha y disminución en la presión sistólica de la arteria pulmonar, en mayor grado que en pacientes que no recibieron una reparación en forma concomitante $24,25,26,27$. Después de conocer los resultados de nuestro hospital, y a la luz de lo publicado por otros centros, hoy en día podemos decir que la IT funcional no es una entidad benigna como se pensaba antes. Es más, ha demostrado ser un factor predictor de menor supervivencia independiente de la HTP o de la disfunción ventricular izquierda ${ }^{28}$. Adicionalmente, se ha demostrado que es un factor de riesgo independiente de malos resultados funcionales y sobre la supervivencia post cirugía valvular mitral ${ }^{29}$. Los hallazgos encontrados en nuestro estudio son consistentes con otros, que han comunicado una mejoría en la función ventricular derecha en cirugías en las que se ha efectuado

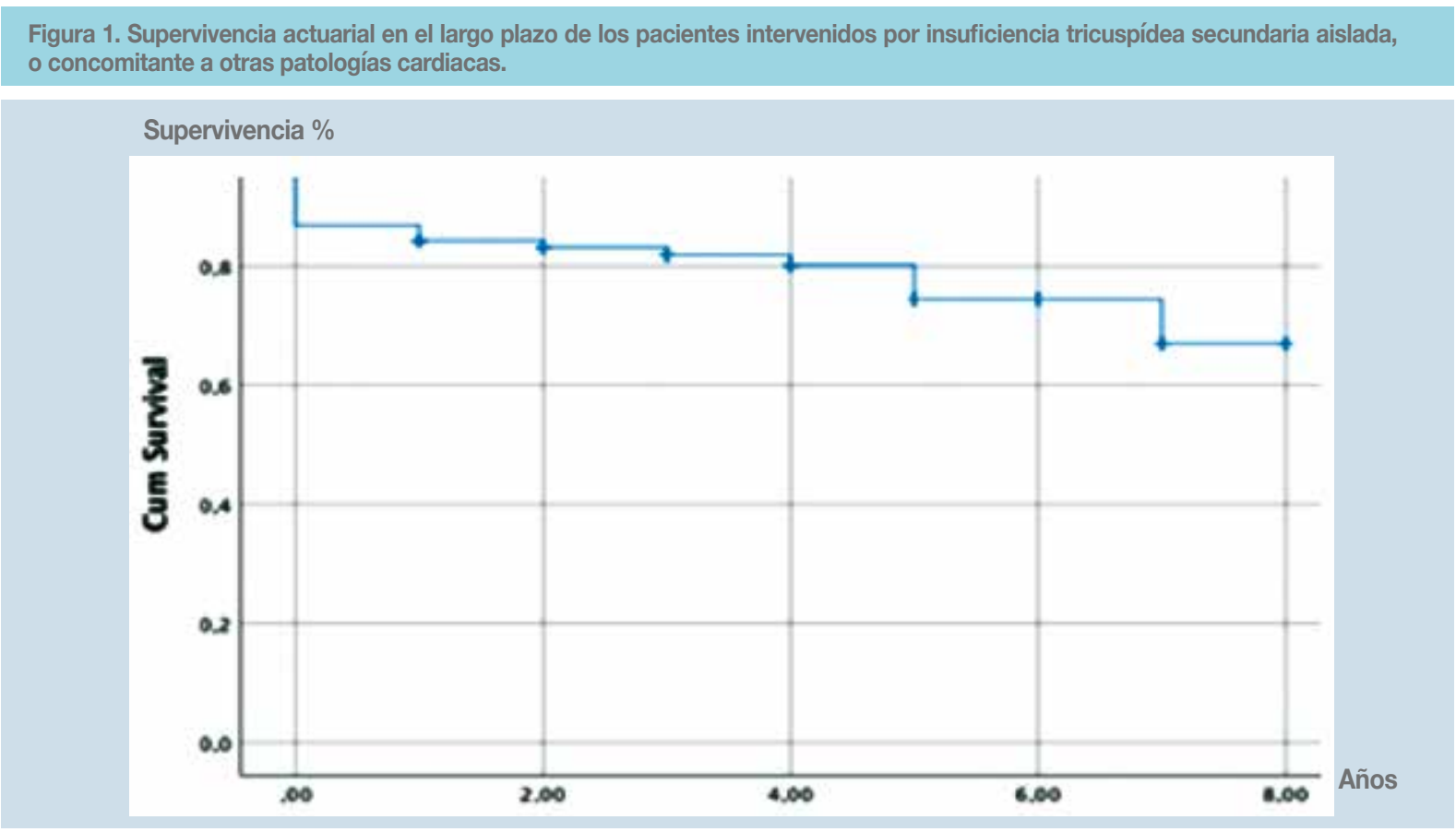


una reparación tricuspídea en forma concomitante 30,31 . La IT produce alteraciones progresivas en la función y estructura de la aurícula y ventrículo derechos ${ }^{6,9,14,24}$, $30,31,32$, lo que puede explicar por qué la estrategia quirúrgica de reparación tricuspídea, diseñada para eliminar la IT importante, ha resultado en una mejoría a largo plazo en la remodelación auricular y ventricular, y confirma lo recomendado por las guías clínicas actuales sobre valvulopatías ${ }^{4,5}$.

La principal limitante de este estudio es su carácter retrospectivo. Por lo mismo, no da cuenta de otras variables como la protección miocárdica utilizada o la ejecución e interpretación de diferentes ecocardiogramas efectuados por diferentes operadores en nuestra institución y en otros centros derivadores, el que no pudo ser efectuado en la totalidad de los pacientes debido a ruralidad extrema o imposibilidad de citar a control ecocardiográfico antes del cierre del estudio. Un elemento importante se- ñalado en diferentes estudios es que, en pacientes con factores de riesgo de IT post operatoria como la presencia de fibrilación auricular, hipertensión pulmonar o disfunción ventricular derecha, la evaluación ecocardiográfica intraoperatoria es de suma importancia en estos pacientes antes de comenzar la cirugía ${ }^{32,33}$.

A pesar de lo señalado, nuestro estudio provee información en el medio nacional que apoya los beneficios de eliminar la IT funcional en pacientes que se operan enfermedades valvulares izquierdas y por otras causas. En conclusión, este estudio apoya fuertemente la estrategia de efectuar de rutina y en forma liberal una anuloplastia tricuspídea en pacientes que presentan IT moderada o severa o con dilatación anular, en forma concomitante con otras patologías cardiacas, Ello, sin consecuencias clínicas adversas y con una excelente evidencia de mejoría en la función ventricular derecha en el largo plazo $^{7,10,14,24,26}$.

\section{Referencias}

1. TARAMASSO M, VANERMEN H, MAISANO F, GUIDOTTI A, LA CANNA G, ALFIERI O. The growing clinical importance of secondary tricuspid regurgitation. J Am Coll Cardiol. 2012;59:703-710. Mylotte D. The forgotten valve no more. EuroIntervention. 2017;12:e1799-e1801.

2. DO QB, PELLERIN M, CARRIER M, CARTIER R, HEBERT Y, PAGE P, et al. Clinical outcome after isolated tricuspid valve replacement: 20-year experience. Can J Cardiol. 2000;16:489-493.

3. DE VEGA NG. Selective, adjustable and permanent annuloplasty. An original technic for the treatment of tricuspid Insufficiency. Rev Esp Cardiol. 1972;25:555-6.

4. BAUMGARTNER H, FALK V, BAX J, DE BONIS M,
HAMM C, HOLM P, et al. 2017 ESC/EACTS Guidelines for the management of valvular heart disease. The Task Force for the Management of Valvular Heart Disease of the European Society of Cardiology (ESC) and the European Association for Cardio-Thoracic Surgery (EACTS) European Heart Journal 2017; 38: 2739-2791

5. NISHIMURA RA, OTTO CM, BONOW RO, CARABELLO BA, ERWIN JP III, GUYTON RA, et al. American College of Cardiology/American Heart Association Task Force on Practice Guidelines. 2014 AHA/ACC guideline for the management of patients with valvular heart disease: a report of the American College of Cardiology/American Heart Association Task Force on Practice Guidelines. J Am Coll Cardiol. 2014;63:e57-e185.

6. TORNOS MAS P, RODRÍGUEZ-PALOMARES JF, ANTU- 
NES MJ. Secondary tricuspid valve regurgitation: a forgotten entity. Heart. 2015;101:1840-1848.

7. NATH J, FOSTER E, HEIDENREICH PA. Impact of tricuspid regurgitation on long-term survival. J Am Coll Cardiol. 2004;43:405-409.

8. KALBACHER D, SCHAFER U, VON BARDELEBEN RS, ZUERN CS, BEKEREDJIAN R, OUARRAK T, et al. Impact of tricuspid valve regurgitation in surgical high-risk patients undergoing MitraClip implantation: results from the TRAMI registry. EuroIntervention. 2017; 12: e1809-e1816.

9. DAHOU A, MAGNE J, CLAVEL MA, CAPOULADE R, BARTKO PE, BERGLER-KLEIN J, et al. Tricuspid regurgitation is associated with increased risk of mortality in patients with lowflow low-gradient aortic stenosis and reduced ejection fraction: results of the multicenter TOPAS study (True or Pseudo-Severe Aortic Stenosis). JACC Cardiovasc Interv. 2015; 8: 588-596.

10. LINDMAN BR, MANIAR HS, JABER WA, LERAKIS S, MACK MJ, SURI RM, et al. Effect of tricuspid regurgitation and the right heart on survival after transcatheter aortic valve replacement: insights from the Placement of Aortic Transcatheter Valves II inoperable cohort. Circ Cardiovasc Interv. 2015; 8: e002073.

11. VASSILEVA CM, SHABOSKY J, BOLEY T, MARKWELL S, HAZELRIGG S. Tricuspid valve surgery: the past 10 years from the Nationwide Inpatient Sample (NIS) database. J Thorac Cardiovasc Surg. 2012; 143: 1043-1049.

12. NISHIMURA RA, OTTO CM, BONOW RO, CARABELLO BA, ERWIN JP III, GUYTON RA, et al. American College of Cardiology/American Heart Association Task Force on Practice Guidelines . 2014 AHA/ACC guideline for the management of patients with valvular heart disease: a report of the American College of Cardiology/American Heart Association Task Force on Practice Guidelines. J Am Coll Cardiol. 2014;63:e57-e185.

13. MORACA RJ, MOON MR, LAWTON JS, GUTHRIE TJ, AUBUCHON KA, MOAZAMI N, et al. Outcomes of tricuspid valve repair and replacement: a propensity analysis. Ann Thorac Surg. 2009; 87: 83-89.

14. KILIC A, SAHA-CHAUDHURI P, RANKIN JS, CONTE JV. Trends and outcomes of tricuspid valve surgery in North America: an analysis of more than 50,000 patients from the Society of Thoracic Surgeons database. Ann Thorac Surg. 2013; 96: 1546-1552.

15. MARQUIS-GRAVEL G, BOUCHARD D, PERRAULT LP, PAGE P, JEANMART H, DEMERS P, et al. Retrospective cohort analysis of 926 tricuspid valve surgeries: clinical and hemodynamic outcomes with propensity score analysis. Am
Heart J. 2012; 163: 851-858.e1.

16. ANDELL P, LI X, MARTINSSON A, ANDERSSON C, STAGMO M, ZOLLER B, SUNDQUIST K, et al. Epidemiology of valvular heart disease in a Swedish nationwide hospital-based register study. Heart. 2017; 103: 1696-1703.

17. PERLMAN G, PRAZ F, PURI R, OFEK H, YE J, PHILIPPON $F$, et al. Transcatheter tricuspid valve repair with a new transcatheter coaptation system for the treatment of severe tricuspid regurgitation: 1-year clinical and echocardiographic results. JACC Cardiovasc Interv. 2017; 10: 1994-2003.

18. POZZOLI A, TARAMASSO M, ZUBER M, MAISANO F. Transcatheter tricuspid valve repair with MitraClip system using intracardiac echocardiography: proof of concept. EuroIntervention. 2017; 13: e1047-e1057.

19. HAHN RT, MEDURI CU, DAVIDSON CJ, LIM S, NAZIF TM, RICCIARDI MJ, et al. Early feasibility study of a transcatheter tricuspid valve annuloplasty: SCOUT Trial 30-day results. J Am Coll Cardiol. 2017; 69: 1795-1806.

20. NICKENIG G, KOWALSKI M, HAUSLEITER J, BRAUN D, SCHOFER J, YZEIRAJ E, et al. Transcatheter treatment of severe tricuspid regurgitation with the edge-to-edge MitraClip technique. Circulation. 2017; 135: 1802-1814.

21. KUWATA S, TARAMASSO M, NIETLISPACH F, MAISANO F. Transcatheter tricuspid valve repair toward a surgical standard: first-in-man report of direct annuloplasty with a cardioband device to treat severe functional tricuspid regurgitation. Eur Heart J. 2017; 38: 1261.

22. LATIB A, MANGIERI A. Transcatheter tricuspid valve repair: new valve, new opportunities, new challenges. J Am Coll Cardiol. 2017; 69: 1807-1810.

23. STUGE O, LIDDICOAT J. Emerging opportunities for cardiac surgeons within structural heart disease. J Thorac Cardiovasc Surg. 2006; 132: 1258-1261.

24. KOELLING TM, AARONSON KD, CODY RJ, BACH DS, ARMSTRONG WF. Prognostic significance of mitral regurgitation and tricuspid regurgitation in patients with left ventricular systolic dysfunction. Am Heart J. 2002; 144: 524-529.

25. BARBANTI M, BINDER RK, DVIR D, TAN J, FREEMAN M, THOMPSON CR, et al. Prevalence and impact of preoperative moderate/severe tricuspid regurgitation on patients undergoing transcatheter aortic valve replacement. Catheter Cardiovasc Interv. 2015; 85: 677-684.

26. NEUHOLD S, HUELSMANN M, PERNICKA E, GRAF A, BONDERMAN D, ADLBRECHT C, et al. Impact of tricuspid 
regurgitation on survival in patients with chronic heart failure: unexpected findings of a long-term observational study. Eur Heart J. 2013; 34: 844-852.

27. LEE JW, SONG JM, PARK JP, LEE JW, KANG DH, SONG JK. Long-term prognosis of isolated significant tricuspid regurgitation. Circ J. 2010; 74: 375-380.

28. TOPILSKY Y, NKOMO VT, VATURY O, MICHELENA HI, LETOURNEAU T, SURI RM, et al. Clinical outcome of isolated tricuspid regurgitation. JACC Cardiovasc Imaging. 2014; 7: 1185-1194.

29. DE MEESTER P, DE COCK D, VAN DE BRUAENE A, GABRIELS C, BUYS R, HELSEN F, et al. Additional tricuspid annuloplasty in mitral valve surgery results in better clinical outcome. Heart. 2015; 101: 720-726.

30. GAMMIE JS, SHENG S, GRIFFITH BP, PETERSON ED, RANKIN JS, O'BRIEN SM, et al. Trends in mitral valve sur- gery in the United States: results from the Society of Thoracic Surgeons Adult Cardiac Surgery Database. Ann Thorac Surg. 2009; 87: 1431-1437- 1439.

31. BADHWAR V, PETERSON ED, JACOBS JP, HE X, BRENNAN JM, O'BRIEN SM, et al. Longitudinal outcome of isolated mitral repair in older patients: results from 14,604 procedures performed from 1991 to 2007. Ann Thorac Surg. 2012; 94 : 1870-1879.

32. JOKINEN JJ, TURPEINEN AK, PITKANEN O, HIPPELAINEN MJ, HARTIKAINEN JE. Pacemaker therapy after tricuspid valve operations: implications on mortality, morbidity, and quality of life. Ann Thorac Surg. 2009; 87: 1806-1814.

33. JOUAN J, MELE A, FLORENS E, CHATELLIER G, CARPENTIER A, ACHOUH P, et al. Conduction disorders after tricuspid annuloplasty with mitral valve surgery: implications for earlier tricuspid intervention. J Thorac Cardiovasc Surg. 2016; 151: 99-103. 